\title{
Non-universality of string tension ratios and gluon confinement at finite temperature
}

\section{Peter N. Meisinger}

Washington University

E-mail: pnmephysics.wustl.edu

Michael C. Ogilvie ${ }^{* \dagger}$

Washington University

E-mail: mco@physics.wustl.edu

\begin{abstract}
We show that the Casimir scaling of string tensions observed in lattice simulations is naturally related to the smallness of the adjoint Polyakov loop in the confined phase. Particles in zero $\mathrm{N}$-ality representations of SU(N) can be used to modify simultaneously the adjoint Polyakov loop expectation value, string tension ratios, and mixing between representations of the same $\mathrm{N}$-ality. This leads to a large class of effective Polyakov loop models, all confining at low temperatures. One limiting case is a $\mathrm{Z}(\mathrm{N})$ spin model, while another is closely related to the deconfining transition in large-N models
\end{abstract}

XXIIIrd International Symposium on Lattice Field Theory

25-30 July 2005

Trinity College, Dublin, Ireland

\footnotetext{
${ }^{*}$ Speaker.

${ }^{\dagger}$ M.C.O. acknowledges the support of the U.S. Dept. of Energy.
} 


\section{Introduction}

There is now a substantial body of lattice simulation results indicating that Casimir scaling [1] or behavior close to it occurs in the string tensions associated with different representations of $S U(N)$ gauge theories in $3+1$ and $2+1$ dimensions. Casimir scaling is exact in two dimensions, but a firm foundation in higher dimensions is lacking. String theory provides a theoretical basis for an alternative, sine-law scaling, but it has proven difficult to differentiate Casimir scaling and sinelaw scaling in simulations. We will show below that this universal, or near-universal, behavior, can be spoiled by terms involving adjoint Polyakov loops added either to the gauge action or to the effective action for Polyakov loops. In addition, such terms also induce large mixing between representations in Polyakov loop two-point functions, and directly control the size of the adjoint Polyakov loop.

The dominant effect on finite-temperature physics of adding heavy partcles in the adjoint representation is well-known. In perturbation theory, the effect on the Polyakov loop $P$ can be modeled by adding a term

$$
-h \operatorname{Tr}_{A} P=-\left[\frac{M^{2} T^{2}}{\pi^{2}} K_{2}(M / T)\right] \operatorname{Tr}_{A} P
$$

to the gauge action with $h>0$. Such terms favor $P \in Z(N)$, and thus spontaneous breaking of $Z(N)$ symmetry. One might believe that only the case $h \geq 0$ is interesting. However, there are several examples where terms arise in the effective action which favor a $Z(N)$-symmetric phase.

Perhaps the most interesting case is recent work by Diakonov et al. [2], who have calculated the contribution to the effective potential of a new class of finite temperature instantons (calorons) with a non-trivial Polyakov loop at spatial infinity. Their results indicate an instability in the deconfined phase at sufficiiently low temperatures. It is very interesting to compare this with related work on $\mathscr{N}=1$ supersymmetric Yang-Mills theory on $R^{3} \times S^{1}$ [3, 4]. String tensions can be calculated exactly from instanton contributions to the effective potential, and show sine-law scaling.

Instability of $P=I$ also occurs in the one-loop effective potential for constant chromomagnetic fields at finite temperature [5]. At low temperatures, the minimum of the real part of the effective potential alternates between the confined and deconfined phases. However, the presence of an imaginary contribution to the effective potential indicates that the Savvidy instability is present at finite temperature.

Another example occurs for gauge theories in small spatial volumes. The integration over global color symmetry leads to a term proportional to the log of Haar measure in the effective action in the form

$$
\sum_{j<k} \ln \left[\sin ^{2}\left(\frac{\theta_{j}-\theta_{k}}{2}\right)\right]=-\sum_{n=1}^{\infty} \frac{1}{n} \operatorname{Tr}_{A}\left[P^{n}+P^{+n}\right]
$$

and this term is responsible for confinement. For all values of $N$, there is a unique set of eigenvalues where Haar measure peaks, corresponding to uniform spacing on the unit circle. For this set of eigenvalues, $\operatorname{Tr}_{R} P=0$ for all representations with non-zero N-ality [6, 7]. For large $N$, there is a phase transition to a deconfining phase [8].

It is thus interesting to consider all values of $h$. In lattice gauge theories, standard arguments [9] tell us that a low-temperature confined phase will exist for all $h$, and there is no phase transition at $\beta=0$ as $h$ is varied. The two limiting cases, $h \rightarrow \pm \infty$, are particularly interesting. As $h$ is 


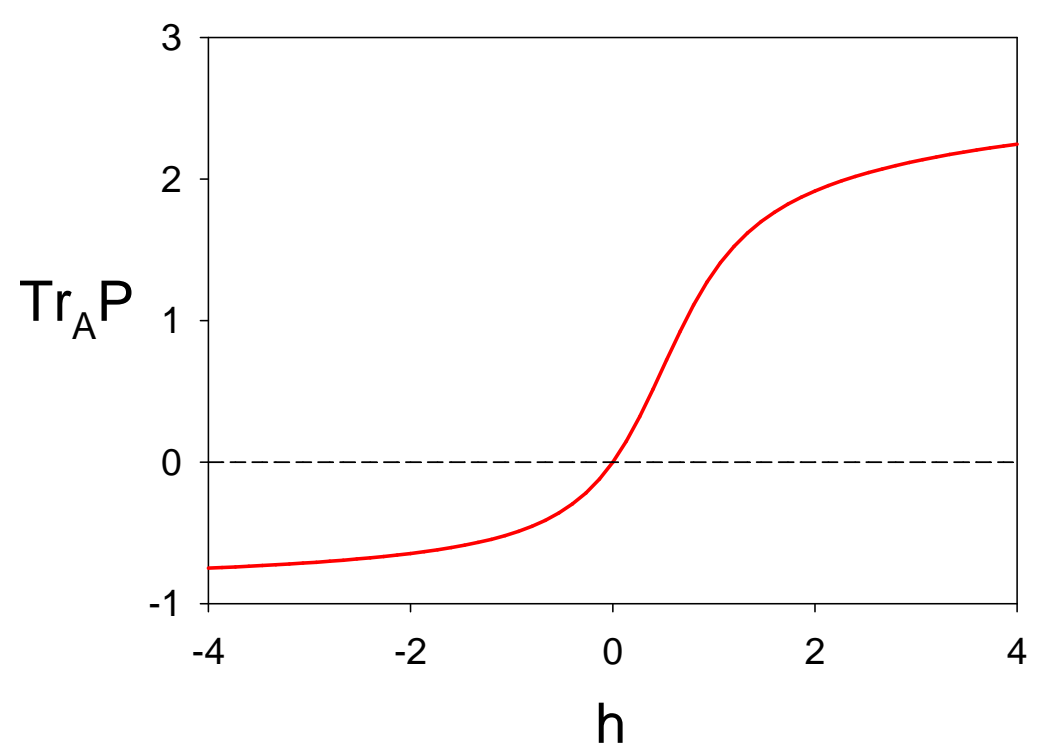

Figure 1: The expected value of $\operatorname{Tr}_{A} P$ as a function of $h$.

taken large and positive, $P$ tends towards elements of $Z(N), P=z^{n}$ with $z=\exp (2 \pi i / N)$, giving $\operatorname{Tr}_{A} P \rightarrow N^{2}-1$. In this limit, the confined phase, with $\operatorname{Tr}_{F} P=0$, must be realized by averaging over different $Z(N)$ values in a manner similar to the high-temperature phase of a $Z(N)$ spin system. We refer to this as the $Z(N)$ limit. As $h$ is taken large and negative, $\operatorname{Tr}_{A} P$ goes to its minimum value of -1 , which leads to $\operatorname{Tr}_{F} P=0$. We refer to this as the master-field limit, because there is a unique set of eigenvalues of $P$, evenly spaced on the unit circle, as occurs in the large-N limit.

\section{1+1 Dimensions}

We consider the case of an external field $h$ coupled to a $(1+1)$-dimensional $S U(2)$ gauge theory. It is convenient to use the Hamiltonian formalism, exchanging the roles of $x$ and $t$, so that the width of the system is $1 / T$. The Hamiltonian can be written as

$$
H=\varepsilon_{0} C_{2}-h \chi_{A}
$$

where $C_{2}$ is the quadratic Casimir operator and the constant $\varepsilon_{0}=g^{2} / 2 T$ will henceforth be set to one. This model is easily studied on a finite-dimensional subspace of the Hilbert space of gaugeinvariant states, which is spanned by the group characters. The energy eigenvalues are related to string tensions by $E_{j}=\sigma_{j} / T$. Figure 1 shows the expectation value $\left\langle\operatorname{Tr}_{A} P\right\rangle$ as a function of $h$. Consistent with our general analysis above, $\left\langle\operatorname{Tr}_{A} P\right\rangle$ asymptotically approches 3 as $h \rightarrow+\infty$, and approaches -1 as $h \rightarrow-\infty$. A special feature of $1+1$ dimensions is that $\left\langle\operatorname{Tr}_{A} P\right\rangle$ is exactly zero when $h=0$; in higher dimensions, $\left\langle\operatorname{Tr}_{A} P\right\rangle$ is very small but positive in the confined phase.

We can define an effective "mass" for each representation $j$ in terms of connected correlation functions as

$$
M_{e f f}^{(j)}(x)=-\frac{d}{d x} \ln \left[\left\langle\chi_{j}(0) \chi_{j}(x)\right\rangle_{c}\right]
$$




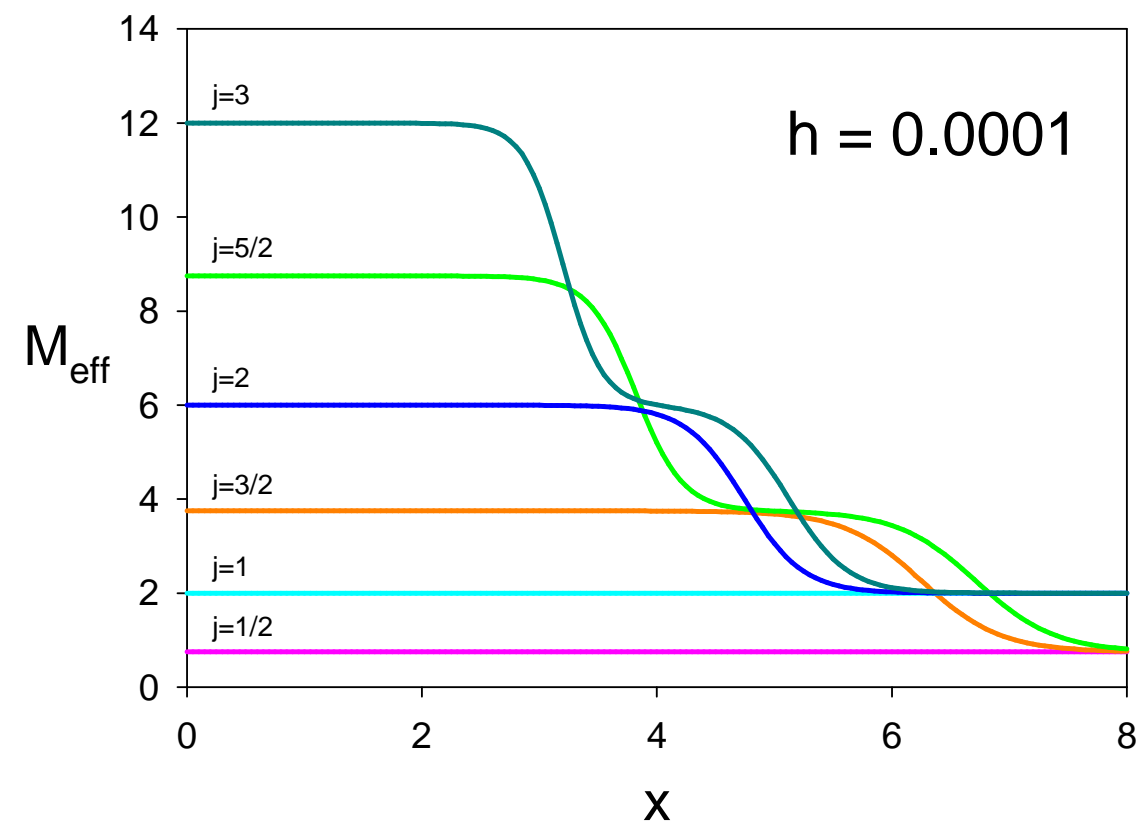

Figure 2: The effective mass $M_{e f f}$ as a function of scaled distance as measured by various Polyakov loop correlation functions.

For large $|h|$, the behavior of the effective masses shows a rapid approach to the lightest string tension in each $Z(2)$ sector. For small values of $h$, we can see the persistence of Casimir scaling over some distance, as shown in figure 2.

Although each correlation function $\left\langle\chi_{j}(0) \chi_{j}(t)\right\rangle_{c}$ is dominated at large distances by the lightest string tension in each $N$-ality sector, the infinite set of string tensions which exist at $h=0$ persist for all $h$, and are obtained from the eigenvalues of $H$ by $E_{j}=\sigma_{j} / T$. For simplicity, we label the eigenvalues by their association with a given representation at $h=0$, i.e., by $j$. As seen in figure 3, eigenvalues, and hence string tensions, vary smoothly with $h$.

The overall picture we have seen in $1+1$ dimensions and $N=2$ should carry over to higher dimensions and larger $N$. In the limit of large positive $h$, Polyakov loops become more and more like $Z(N)$ spins, and averaging over different values of $Z(N)$ is necessary for confinement. In the opposite limit, a single field configuration gives confinement without the need for any averaging.

\section{Higher Dimensions}

We have studied the effects of adjoint potential terms in lattice Polyakov loop effective actions using mean field theory, with results similar to those seen in $1+1$ dimensions. We use the most general $Z(N)$-invariant form for the Polyakov loop effective action

$$
S_{e f f}=-\sum_{j A k B} J_{j A k B} \chi_{j A} \chi_{k B}-\sum_{A j} h_{A j} \chi_{j A}+. .
$$

where A and B label representations and $\mathrm{j}$ and $\mathrm{k}$ label sites. The couplings $h_{j A}$ can be non-zero only for representations of zero $N$-ality. The couplings $J_{j A k B}$ are zero unless $A$ and $B$ have total 


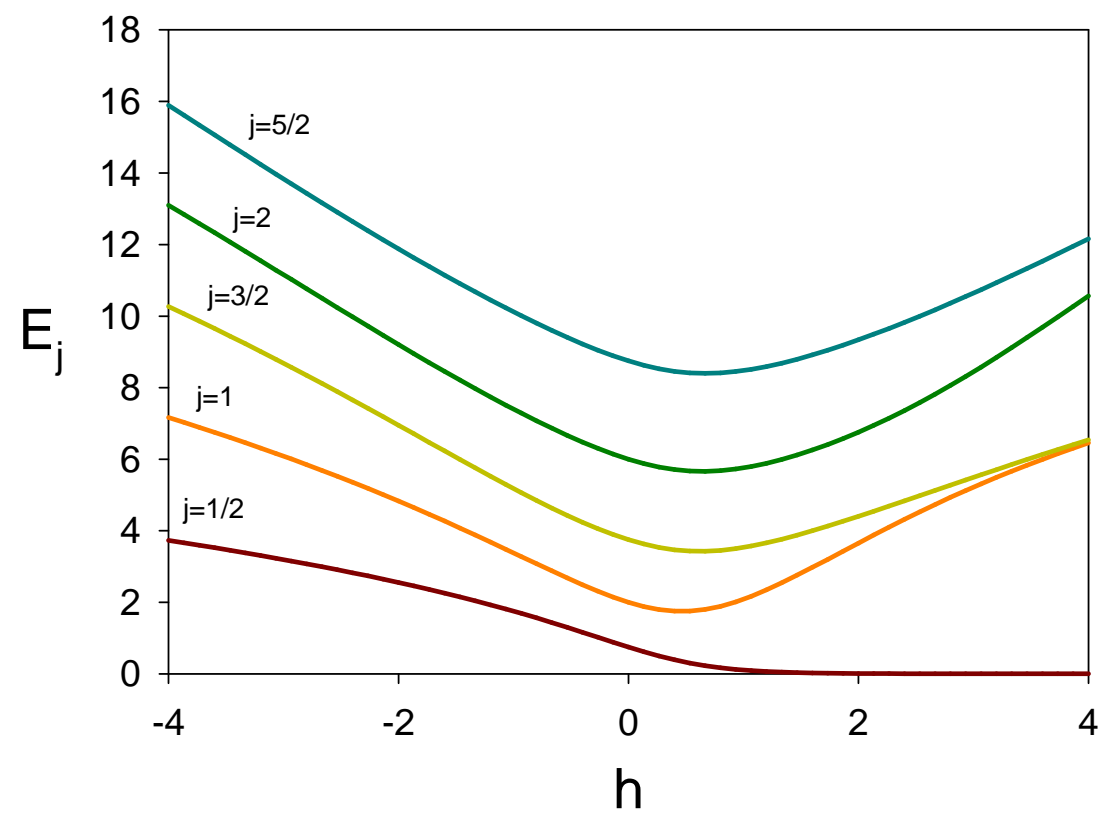

Figure 3: Eigenvalues $E_{j}=\sigma_{j} / T$ of the Hamiltonian as a function of $h$.

$\mathrm{N}$-ality zero, and there may be additional terms involving three or more sites. The dominant term is known from lattice simulations to be a positive two-site nearest-neighbor coupling $[10,11]$. In the strong coupling limit, the coefficients $J_{j A k B}$ can be calculated analytically; the couplings $h_{j A}$ are identically zero in that limit. The mean fields are given by

$$
K_{j A}=\sum_{B k} J_{j A k B} M_{k B}+h_{j A}+. .
$$

and are determined self-consistently in terms of $M_{j B}$ via

$$
M_{j B}=\left\langle\chi_{j B}\right\rangle_{0}=\frac{\int\left(d U_{j}\right) \chi_{j B} \exp \sum_{A} K_{j A} \chi_{j A}}{\int\left(d U_{j}\right) \exp \sum_{A} K_{j A} \chi_{j A}}
$$

where the single-site integral is over the Polyakov loop variable $U_{j}$. The Polyakov loop two-point functions satisfy

$$
\sum_{B k}\left[-J_{j A k B}+\delta_{j k} \frac{\partial K_{j A}}{\partial M_{j B}}\right] G_{k B l C}=\delta_{j l} \delta_{A C}
$$

where possible extra terms have been suppressed.

It is straightforward to show that the phenomena observed in $1+1$ dimensions as the adjoint coupling $h_{A d j}$ is varied occur using mean field theory in higher dimensions as well. The adjoint expectation value $\left\langle\chi_{A d j}\right\rangle_{0}$, an indicator of gluon confinement, varies with $h$, taking its extremal values when $h \rightarrow \pm \infty$. The matrix $\partial K_{j A} / \partial M_{j B}$ is similar to a mass term in continuum field theories. It is the inverse of

$$
\partial M_{j B} / \partial K_{j A}=\left\langle\chi_{j B} \chi_{j A}\right\rangle_{0}-\left\langle\chi_{j B}\right\rangle_{0}\left\langle\chi_{j A}\right\rangle_{0} .
$$

From this, it is easy to show the smooth variation of string tension ratios as $h$ is varied, and the strong mixing of representations of the same $N$-ality when $|h|$ is large. Other features of two- 
dimensions, such as the rapid decrease to zero of the lightest string tension as $h \rightarrow \infty$, also occur in mean field theory.

The small value of the renormalized adjoint Polyakov loop measured in lattice simulations is consistent with all the mean field couplings $K_{j A}$ being small. This would in turn imply that the couplings $h$ are small as well. In the limiting case where all $K$ 's and all $h$ 's are zero, the matrix $\partial K_{j A} / \partial M_{j B}$ is one only when $A$ and $B$ are conjugate representations, and zero otherwise. In this limiting case, mixing can still occur via the two-site coupling $J_{j A k B}$. Such a term has recently been measured in $S U(2)$ lattice simulations, but it is small [12].

\section{References}

[1] L. Del Debbio, M. Faber, J. Greensite and S. Olejnik, Casimir Scaling vs. Abelian Dominance in QCD String Formation, Phys. Rev. D 53, 5891 (1996) [arXiv:hep-lat/9510028].

[2] D. Diakonov, N. Gromov, V. Petrov and S. Slizovskiy, Quantum weights of dyons and of instantons with non-trivial holonomy, Phys. Rev. D 70, 036003 (2004) [arXiv:hep-th/0404042].

[3] N. M. Davies, T. J. Hollowood, V. V. Khoze and M. P. Mattis, Gluino condensate and magnetic monopoles in supersymmetric gluodynamics, Nucl. Phys. B 559, 123 (1999) [arXiv:hep-th/9905015].

[4] N. M. Davies, T. J. Hollowood and V. V. Khoze, Monopoles, affine algebras and the gluino condensate, J. Math. Phys. 44, 3640 (2003) [arXiv:hep-th/0006011].

[5] P. N. Meisinger and M. C. Ogilvie, The finite temperature SU(2) Savvidy model with a non-trivial Polyakov loop, Phys. Rev. D 66, 105006 (2002) [arXiv:hep-ph/0206181].

[6] P. N. Meisinger, T. R. Miller and M. C. Ogilvie, Phenomenological equations of state for the quark-gluon plasma, Phys. Rev. D 65, 034009 (2002) [arXiv:hep-ph/0108009].

[7] P. N. Meisinger, M. C. Ogilvie and T. R. Miller, Gluon quasiparticles and the Polyakov loop, Phys. Lett. B 585, 149 (2004) [arXiv:hep-ph/0312272].

[8] O. Aharony, J. Marsano, S. Minwalla, K. Papadodimas and M. Van Raamsdonk, A first order deconfinement transition in large N Yang-Mills theory on a small S**3, Phys. Rev. D 71, 125018 (2005) [arXiv:hep-th/0502149].

[9] E. H. Fradkin and S. H. Shenker, Phase Diagrams Of Lattice Gauge Theories With Higgs Fields, Phys. Rev. D 19, 3682 (1979).

[10] A. Gocksch and M. Ogilvie, Microcanonical Determination Of Effective Spin Models For Finite Temperature QCD, Phys. Rev. Lett. 54, 1772 (1985).

[11] B. R. Moore and M. Ogilvie, Finite Temperature QCD And SU(3) Spin Systems, Nucl. Phys. Proc. Suppl. 17, 350 (1990).

[12] T. Heinzl, T. Kaestner and A. Wipf, Effective actions for the SU(2) confinement-deconfinement phase transition, Phys. Rev. D 72, 065005 (2005) [arXiv:hep-lat/0502013]. 